\title{
Exact Solutions of the BBM and MBBM Equations by the Generalized (G'/G )-expansion Method Equations
}

\author{
J. Manafianheris
}

Islamic Azad University-Ahar Branch, Ahar, Iran

\begin{abstract}
In this article, we establish exact solutions for the BBM and the MBBM equations by using a generalized $\left(\mathrm{G}^{\prime} / \mathrm{G}\right)$-expansion method. The generalized $\left(\mathrm{G}^{\prime} / \mathrm{G}\right)$-expansion method was used to construct solitary wave and periodic wave solutions of nonlinear evolution equations. This method is developed for searching exact travelling wave solutions of nonlinear partial differential equations. It is shown that the $\left(\mathrm{G}^{\prime} / \mathrm{G}\right)$-expansion method is straightforward and powerful mathematical tool for solving nonlinear problems.
\end{abstract}

Keywords Generalized (G'/G )-Expansion Method, BBM, MBBM Equations, Solitary Wave, Periodic Wave

\section{Introduction}

Nonlinear phenomena play a fundamental role in applied mathematics and physics. Recently, the study of nonlinear partial differential equations in modelling physical phenomena has become an important tool. The investigation of the travelling wave solutions plays an important role in nonlinear sciences. A variety of powerful methods has been presented, such as the inverse scattering transform[1], Hirot a's bilinear method[2], sine-cosine method[3], homotopy perturbation method[4], homotopy analysis method[5, 6], variational iteration method[7, 8, 9], tanh-function method[10], B"acklund transformation[11], Exp-function method $[12,13,14,15,16]$ and so on. The basic idea of the $\left(\mathrm{G}^{\prime} / \mathrm{G}\right)$-expansion method was first proposed by Wang's[17]. A new method is to look for travelling wave solutions of nonlinear evolution equations (NLEEs) and systematically illustrated in Refs.[17, 18, 19, 20]. Zhang et al.[18] have examined the generalized $\left(\mathrm{G}^{\prime} / \mathrm{G}\right)$-expansion method and its applications. Authors of [19] have used to $\mathrm{mKdV}$ equation with variable coefficients using the $\left(\mathrm{G}^{\prime} / \mathrm{G}\right.$ )-expansion method. Also, Bekir[20] has used to application of the $\left(\mathrm{G}^{\prime} / \mathrm{G}\right)$-expansion method for nonlinear evolution equations (NLEEs). In this article, we used the generalized $\left(\mathrm{G}^{\prime} / \mathrm{G}\right)$-ex pansion method to investigate the Benjamin-Bona-Mahony (BBM) equation $[22,23,24]$

$$
u_{t}+u_{x}-a\left(u^{2}\right)_{x}-b u_{x x t}=0,
$$

is well known in physical applications. This equation models long waves in a nonlinear dispersive system, and as a result, the BBM equation incorporates dispersive effects. The

* Corresponding author:

manafeian2@gmail.com (J. Manafianheris)

Published online at http://journal.sapub.org/ijge

Copyright (C) 2012 Scientific \& Academic Publishing. All Rights Reserved solutions of the BBM equation exhibit definite soliton-like behavior that is not explainable by any known theory[21]. Both KdV and BBM equations cover cases of the following type[22]: surface waves of long wavelength in liquids, acoustic-gravity waves in compressible fluids, hydromagnet ics waves in cold plasm, acoustic waves in anharmonic crystals. Motivated by the rich treasure of the Benjamin-Bo na-Mahony equation in science, we will study nonlinear dispersive the modified Benjamin-Bona-Mahony equation (MBBM)[24]

$$
u_{t}+u_{x}+a u^{2} u_{x}+u_{x x x}=0
$$

which was first derived to describe an approximation for surface long waves in nonlinear dispersive media[25]. The equation can also characterize the hydromagnetic waves in cold plasma, acoustic waves in anharmonic crystals and acoustic-gravity waves in compressible fluids[26, 27]. The article is organized as follows: In Section 2, first we briefly give the steps of the method and apply it to solve nonlinear partial differential equations. In Sections 3 and 4, we employ this technique to the BBM and the MBBM equations. Also a conclusion is given in Section 5 .

\section{Basic Idea of $\left(G^{\prime} / G\right)$-expansion Method}

We give the detailed description of method which first presented by Wang[20].

Step 1. For a given nonlinear partial differential equations (NLPDEs) with independent variables $X=(x, y, z, t)$ and dependent variable $u$

$P\left(u, u_{t}, u_{x}, u_{y}, u_{z}, u_{x x}, u_{y y}, u_{z z}, u_{x y}, u_{t t}, u_{t x}, u_{t y}, u_{t z}, \ldots\right)=0$,

can be converted to an ODE

$$
\mathrm{M}\left(\mathrm{u},-\mathrm{c} u^{\prime}, u^{\prime}, u^{\prime}, u^{\prime}, u^{\prime \prime}, \ldots\right) \text {, }
$$


which transformation $\xi=x+y-c t$ is wave variable. Also, $\mathrm{c}$ is constant to be determined later.

Step 2. We seek its solutions in the more general polynomial form as follows

$$
\mathrm{u}(\xi)=a_{0}+\sum_{k=1}^{m} a_{k}\left(\frac{G^{\prime}(\xi)}{G(\xi)}\right)^{k},
$$

where $G(\xi)$ satisfies the second order linear ordinary differential equation (LODE) in the form

$$
G^{\prime \prime}(\xi)+\lambda G^{\prime}(\xi)+\mu G(\xi)=0,
$$

where $a_{0}, a_{k}(\mathrm{k}=1,2, \ldots, \mathrm{m}), \lambda$ and $\mu$ are constants to be determined later, $a_{m}=0$, but the degree of which is generally equal to or less than $m-1$, the positive integer $m$ can be determined by considering the homogeneous balance between the highest order derivatives and nonlinear terms appearing in Eq. (2.2).

Step 3. Substituting (2.3) and Eq. (2.4) into Eq. (2.2) with the value of $m$ obtained in Step 1. Collectingthe coefficients of $\left(\frac{G^{\prime}(\xi)}{G(\xi)}\right)^{k}(\mathrm{k}=1,2, \ldots, \mathrm{m})$, then setting each coefficient to zero, we can get a set of over-determined partial differential equations for $a_{0}, a_{i}(\mathrm{i}=1,2, \ldots, \mathrm{m}), \lambda, \mathrm{c}$ and $\mu$ with the aid of symbolic computation Maple 12.

Step 4. Solving the algebraic equations in Step 3, then substituting $a_{i}, \ldots, a_{m}, c$ and general solutions of Eq. (2.4) into (2.3) we can obtain a series of fundamental solutions of Eq. (2.1) depending of the solution $G(\xi)$ of Eq. (2.4).

\section{The Benjamin-Bona-Mahony (BBM) Equation}

In this section we employ the ( $\left.\mathrm{G}^{\prime} / \mathrm{G}\right)$-expansion method to the BBM equation as follows

$$
u_{t}+u_{x}-a\left(u^{2}\right)_{x}-b u_{x x t}=0,
$$

and by using the wave variable $\xi=x-c t$ reduces it to an ODE

$$
-c u+u-a u^{2}+b c u^{\prime \prime}=0,
$$

obtained by integrating the resulting equation and by considering each constant of integration to be zero. In order to determine value of $m$, we balance the linear term of the highest order $u^{\prime \prime}$ with the highest order nonlinear term $u^{2}$ in Eq. (3.2), along with (2.3) we get

$$
\begin{gathered}
u^{2}(\xi)=a_{m}^{2}\left(\frac{G^{\prime}(\xi)}{G(\xi)}\right)^{2 m}+\cdots, \\
u_{\xi}(\xi)=-m a_{m}\left(\frac{G^{\prime}(\xi)}{G(\xi)}\right)^{m+1}+\cdots, \\
u_{\xi \xi}(\xi)=m(m+1) a_{m}\left(\frac{G^{\prime}(\xi)}{G(\xi)}\right)^{m+2}+\cdots
\end{gathered}
$$

Balancing $u^{\prime \prime}$ with $u^{2}$ in Eq. (3.2), based on (3.3) we required that $2 m=m+2 \Rightarrow m=2$. We can suppose that the solutions of Eq. (3.1) is as follows

$$
u(\xi)=a_{0}+a_{1}\left(\frac{G^{\prime}(\xi)}{G(\xi)}\right)+a_{2}\left(\frac{G^{\prime}(\xi)}{G(\xi)}\right)^{2}, \quad a_{2} \neq 0 .
$$

and therefore

$$
\begin{aligned}
& u^{2}(\xi)=a_{2}{ }^{2}\left(\frac{G^{\prime}(\xi)}{G(\xi)}\right)^{4}+2 a_{1} a_{2}\left(\frac{G^{\prime}(\xi)}{G(\xi)}\right)^{3}+\left(a_{1}{ }^{2}+2 a_{0} a_{2}\right)\left(\frac{G^{\prime}(\xi)}{G(\xi)}\right)^{2}+2 a_{0} a_{1}\left(\frac{G^{\prime}(\xi)}{G(\xi)}\right) \\
& +a_{0}^{2} \\
& u_{\xi \xi}(\xi)=6 a_{2}\left(\frac{G^{\prime}(\xi)}{G(\xi)}\right)^{4}+\left(2 a_{1}+10 a_{2} \lambda\right)\left(\frac{G^{\prime}(\xi)}{G(\xi)}\right)^{3}+\left(8 a_{2} \mu+3 a_{1} \lambda+4 a_{2} \lambda^{2}\right)\left(\frac{G^{\prime}(\xi)}{G(\xi)}\right)^{2} \\
& +\left(6 a_{2} \lambda \mu+2 a_{1} \mu+a_{1} \lambda^{2}\right)\left(\frac{G^{\prime}(\xi)}{G(\xi)}\right)+2 a_{2} \mu^{2}+a_{1} \lambda \mu .
\end{aligned}
$$

Substituting (3.4)-(3.6) in Eq. (3.3), and by using the well-known Maple software, we obtain the system of the following results

$$
a_{0}=\frac{b c\left(\lambda^{2}+2 \mu\right)}{a}, \quad a_{1}=\frac{6 b c \lambda}{a}, \quad a_{2}=\frac{6 b c}{a}, \quad c=\frac{1}{1+b\left(\lambda^{2}-4 \mu\right)},
$$

or

$$
a_{0}=\frac{6 b \mu}{a}, \quad a_{1}=\frac{6 b c \lambda}{a}, \quad a_{2}=\frac{6 b c}{a}, \quad c=\frac{1}{1-b\left(\lambda^{2}-4 \mu\right)},
$$

where $\lambda$ and $\mu$ are arbitrary constants. Substituting (3.7) and (3.8) into expression (3.4), can be written as

$$
\begin{gathered}
u(\xi)=\frac{b\left(\lambda^{2}+2 \mu\right)}{1+b\left(\lambda^{2}-4 \mu\right)}+\frac{6 b \lambda}{1+b\left(\lambda^{2}-4 \mu\right)}\left(\frac{G^{\prime}(\xi)}{G(\xi)}\right)+\frac{6 b}{1+b\left(\lambda^{2}-4 \mu\right)}\left(\frac{G^{\prime}(\xi)}{G(\xi)}\right)^{2}, \\
\xi=x-\frac{1}{1+b\left(\lambda^{2}-4 \mu\right)} t, \\
u(\xi)=\frac{6 b \mu}{1-b\left(\lambda^{2}-4 \mu\right)}+\frac{6 b \lambda}{1-b\left(\lambda^{2}-4 \mu\right)}\left(\frac{G^{\prime}(\xi)}{G(\xi)}\right)+\frac{6 b}{1-b\left(\lambda^{2}-4 \mu\right)}\left(\frac{G^{\prime}(\xi)}{G(\xi)}\right)^{2}, 3
\end{gathered}
$$

or

$$
\xi=x-\frac{1}{1-b\left(\lambda^{2}-4 \mu\right)} t .
$$

Substituting the general solutions of Eq. (2.4) into (3.9) and (3.10) we have three types of exact solutions of Eq. (3.1). I. When $\lambda^{2}-4 \mu>0$, we obtain hyperbolic function solution

$$
\begin{aligned}
& u_{1}(\xi)=\frac{3}{2} \frac{b\left(\lambda^{2}-4 \mu\right)}{a+a b\left(\lambda^{2}-4 \mu\right)}\left(\frac{c_{1} \sinh \left(\frac{\sqrt{\lambda^{2}-4 \mu} \xi}{2}\right)+C_{2} \cosh \left(\frac{\sqrt{\lambda^{2}-4 \mu} \xi}{2}\right)}{C_{1} \cosh \left(\frac{\sqrt{\lambda^{2}-4 \mu} \xi}{2}\right)+C_{2} \sinh \left(\frac{\sqrt{\lambda^{2}-4 \mu \xi}}{2}\right)}\right)^{2} \\
& -\frac{1}{2} \frac{b\left(\lambda^{2}-4 \mu\right)}{a+a b\left(\lambda^{2}-4 \mu\right)}, \quad \xi=x-\frac{1}{1+b\left(\lambda^{2}-4 \mu\right)} t, \\
& u_{2}(\xi)=\frac{3}{2} \frac{b\left(\lambda^{2}-4 \mu\right)}{a-a b\left(\lambda^{2}-4 \mu\right)}\left(\frac{C_{1} \sinh \left(\frac{\sqrt{\lambda^{2}-4 \mu} \xi}{2}\right)+C_{2} \cosh \left(\frac{\sqrt{\lambda^{2}-4 \mu} \xi}{2}\right)}{C_{1} \cosh \left(\frac{\sqrt{\lambda^{2}-4 \mu} \xi}{2}\right)+C_{2} \sinh \left(\frac{\sqrt{\lambda^{2}-4 \mu} \xi}{2}\right)}\right)^{2} 3 \\
& -\frac{3}{2} \frac{b\left(\lambda^{2}-4 \mu\right)}{a-a b\left(\lambda^{2}-4 \mu\right)}, \quad \xi=x-\frac{1}{1-b\left(\lambda^{2}-4 \mu\right)} t .
\end{aligned}
$$

II. When $\lambda^{2}-4 \mu<0$, we have trigonometric function solution

$$
\begin{gathered}
u_{3}(\xi)=\frac{3}{2} \frac{b\left(4 \mu-\lambda^{2}\right)}{a+a b\left(\lambda^{2}-4 \mu\right)}\left(\frac{-C_{1} \sin \left(\frac{\sqrt{4 \mu-\lambda^{2}} \xi}{2}\right)+C_{2} \cos \left(\frac{\sqrt{4 \mu-\lambda^{2}} \xi}{2}\right)}{C_{1} \cos \left(\frac{\sqrt{4 \mu-\lambda^{2}} \xi}{2}\right)+C_{2} \sin \left(\frac{\sqrt{4 \mu-\lambda^{2} \xi}}{2}\right)}\right)^{2} \\
-\frac{1}{2} \frac{b\left(\lambda^{2}-4 \mu\right)}{a+a b\left(\lambda^{2}-4 \mu\right)}, \quad \xi=x-\frac{1}{1+b\left(\lambda^{2}-4 \mu\right)} t,
\end{gathered}
$$




$$
\begin{gathered}
u_{4}(\xi)=\frac{3}{2} \frac{b\left(4 \mu-\lambda^{2}\right)}{a-a b\left(\lambda^{2}-4 \mu\right)}\left(\frac{-C_{1} \sin \left(\frac{\sqrt{4 \mu-\lambda^{2}} \xi}{2}\right)+C_{2} \cos \left(\frac{\sqrt{4 \mu-\lambda^{2}} \xi}{2}\right)}{C_{1} \cos \left(\frac{\sqrt{4 \mu-\lambda^{2}} \xi}{2}\right)+C_{2} \sin \left(\frac{\sqrt{4 \mu-\lambda^{2}} \xi}{2}\right)}\right)^{2} \\
-\frac{3}{2} \frac{b\left(\lambda^{2}-4 \mu\right)}{a-a b\left(\lambda^{2}-4 \mu\right)}, \quad \xi=x-\frac{1}{1-b\left(\lambda^{2}-4 \mu\right)} t .
\end{gathered}
$$

III. When $\lambda^{2}-4 \mu=0$, we get rational solution

$$
u_{5}(\xi)=\frac{6 b}{a} \frac{C_{2}^{2}}{\left(C_{1}+C_{2} \xi\right)^{2}}, \quad \xi=x-t .
$$

(I) The first set: If $C_{1} \neq 0, C_{2}=0, \lambda>0, \mu=0$, then solution (3.11) and (3.12), respectively, give:

$$
\begin{aligned}
& u_{6}(\mathrm{x}, \mathrm{t})=\frac{b \lambda^{2}}{2 a+2 a b \lambda^{2}}\left\{3 \tanh ^{2}\left[\frac{\lambda}{2}\left(x-\frac{1}{1+b \lambda^{2}} t\right)\right]-1\right\}, \\
& u_{7}(\mathrm{x}, \mathrm{t})=-\frac{3 b \lambda^{2}}{2 a-2 a b \lambda^{2}} \operatorname{sech}^{2}\left[\frac{\lambda}{2}\left(x-\frac{1}{1-b \lambda^{2}} t\right)\right] .
\end{aligned}
$$

Also, if $C_{1}=0, C_{2} \neq 0, \lambda>0, \mu=0$, then (3.11) and (3.12) give respectively

$$
\begin{aligned}
& u_{8}(\mathrm{x}, \mathrm{t})=\frac{b \lambda^{2}}{2 a+2 a b \lambda^{2}}\left\{3 \operatorname{coth}^{2}\left[\frac{\lambda}{2}\left(x-\frac{1}{1+b \lambda^{2}} t\right)\right]-1\right\} \\
& u_{9}(\mathrm{x}, \mathrm{t})=\frac{3 b \lambda^{2}}{2 a-2 a b \lambda^{2}} \operatorname{csch}^{2}\left[\frac{\lambda}{2}\left(x-\frac{1}{1-b \lambda^{2}} t\right)\right] .
\end{aligned}
$$

(II) The second set: If $C_{1} \neq 0, C_{2}=0, \lambda=0, \mu>0$, then (3.13) and (3.14) can be written as respectively

$$
\begin{aligned}
& u_{10}(\mathrm{x}, \mathrm{t})=\frac{2 b \mu}{a-4 a b \mu}\left\{3 \tan ^{2}\left[\sqrt{\mu}\left(x-\frac{1}{1-4 b \mu} t\right)\right]+1\right\}, \\
& u_{11}(\mathrm{x}, \mathrm{t})=\frac{6 b \mu}{a+4 a b \mu} \sec ^{2}\left[\sqrt{\mu}\left(x-\frac{1}{1+4 b \mu} t\right)\right] .
\end{aligned}
$$

In particular, if $C_{1}=0, C_{2} \neq 0, \lambda=0, \mu>0$, then (3.13) and (3.14) can be written as respectively

$$
\begin{aligned}
& u_{12}(\mathrm{x}, \mathrm{t})=\frac{2 b \mu}{a-4 a b \mu}\left\{3 \cot ^{2}\left[\sqrt{\mu}\left(x-\frac{1}{1-4 b \mu} t\right)\right]+1\right\}, \\
& u_{13}(\mathrm{x}, \mathrm{t})=\frac{6 b \mu}{a+4 a b \mu} \csc ^{2}\left[\sqrt{\mu}\left(x-\frac{1}{1+4 b \mu} t\right)\right] .
\end{aligned}
$$

(III) The third set: If $C_{1} \neq 0, C_{2}=0, \lambda=0, \mu<0$, then (3.11) and (3.12) give respectively

$$
\begin{aligned}
& u_{14}(\mathrm{x}, \mathrm{t})=\frac{2 b \mu}{a-4 a b \mu}\left\{2-3 \tanh ^{2}\left[\sqrt{-\mu}\left(x-\frac{1}{1-4 b \mu} t\right)\right]\right\}, \\
& u_{15}(\mathrm{x}, \mathrm{t})=\frac{6 b \mu}{a+4 a b \mu} \operatorname{sech}^{2}\left[\sqrt{-\mu}\left(x-\frac{1}{1+4 b \mu} t\right)\right] .
\end{aligned}
$$

But, if $C_{1}=0, C_{2} \neq 0, \lambda=0, \mu<0$, then (3.11) and (3.12) give respectively

$$
\begin{aligned}
& u_{16}(\mathrm{x}, \mathrm{t})=\frac{2 b \mu}{a-4 a b \mu}\left\{2-3 \operatorname{coth}^{2}\left[\sqrt{-\mu}\left(x-\frac{1}{1-4 b \mu} t\right)\right]\right\}, \\
& u_{17}(\mathrm{x}, \mathrm{t})=\frac{6 b \mu}{a+4 a b \mu} \operatorname{csch}^{2}\left[\sqrt{-\mu}\left(x-\frac{1}{1+4 b \mu} t\right)\right] .
\end{aligned}
$$

\section{The Modified \\ Benjamin-Bona-Mahony (MBBM) Equation}

We cosider the $\left(G^{\prime} / G\right)$-expansion method to the modified
Benjamin-Bona-Mahony (MBBM) as follows

$$
u_{t}+u_{x}+a u^{2} u_{x}+u_{x x x}=0 \text {, }
$$

and by using the wave variable $\xi=x-c t$ reduces it to an ODE

$$
-c u+u+\frac{a}{3} u^{3}+u^{\prime \prime}=0,
$$

obtained by integrating the resulting equation and by considering each constant of integration to be zero. Balancing the linear term of the highest order $u^{\prime \prime}$ with the highest order nonlinear term $u^{3}$ in Eq. (4.2), and by using Eq. (2.3) we will have

$$
\begin{gathered}
u^{3}(\xi)=a_{m}{ }^{3}\left(\frac{G^{\prime}(\xi)}{G(\xi)}\right)^{3 m}+\cdots, \\
u_{\xi}(\xi)=-m a_{m}\left(\frac{G^{\prime}(\xi)}{G(\xi)}\right)^{m+1}+\cdots, \\
u_{\xi \xi}(\xi)=m(m+1) a_{m}\left(\frac{G^{\prime}(\xi)}{G(\xi)}\right)^{m+2}+\cdots .
\end{gathered}
$$

Balancing $u^{\prime \prime}$ with $u^{3}$ in Eq. (4.2), based on (4.3) we required that $3 m=m+2 \Rightarrow m=1$. We can suppose that the solutions of Eq. (4.1) is as follows

$$
u(\xi)=a_{0}+a_{1}\left(\frac{G^{\prime}(\xi)}{G(\xi)}\right), \quad a_{1} \neq 0,
$$

and therefore

$$
\begin{aligned}
& u^{3}(\xi)=a_{1}{ }^{3}\left(\frac{G^{\prime}(\xi)}{G(\xi)}\right)^{3}+3 a_{1}{ }^{2} a_{0}\left(\frac{G^{\prime}(\xi)}{G(\xi)}\right)^{2}+3 a_{0}{ }^{2} a_{1}\left(\frac{G^{\prime}(\xi)}{G(\xi)}\right)+a_{0}{ }^{3},(4.5) \\
& \left.u_{\xi \xi}(\xi)=2 a_{1}\left(\frac{G^{\prime}(\xi)}{G(\xi)}\right)^{3}+3 a_{1} \lambda\left(\frac{G^{\prime}(\xi)}{G(\xi)}\right)^{2}+\left(2 a_{1} \mu+a_{1} \lambda^{2}\right)\left(\frac{G^{\prime}(\xi)}{G(\xi)}\right)+a_{1} \lambda \mu .4 .6\right)
\end{aligned}
$$

Substituting (4.4)-(4.6) in Eq. (4.2), and by using the well-known Maple software, we will have

$$
a_{0}= \pm \frac{3 \lambda}{\sqrt{6 a}} i, \quad a_{1}= \pm \sqrt{\frac{6}{a}} i, \quad c=1-\frac{\lambda^{2}-4 \mu}{2},
$$

where $\lambda$ and $\mu$ are arbitrary constants. Substituting (4.7) into expression (4.4), can be written as

$$
u(\xi)= \pm \frac{3 \lambda}{\sqrt{6 a}} i+ \pm \sqrt{\frac{6}{a}} i\left(\frac{G^{\prime}(\xi)}{G(\xi)}\right), \quad \xi=x-\frac{2-\lambda^{2}+4 \mu}{2} t .
$$

Substituting the general solutions of Eq. (2.4) into (4.8) we have three types of exact solutions of Eq. (4.1). I. When $\lambda^{2}-4 \mu>0$ we obtain hyperbolic function solution

$$
\begin{aligned}
& u_{1}(\xi)= \pm \sqrt{\frac{3\left(\lambda^{2}-4 \mu\right)}{2 a}} i\left(\frac{C_{1} \sinh \left(\frac{\sqrt{\lambda^{2}-4 \mu} \xi}{2}\right)+C_{2} \cosh \left(\frac{\sqrt{\lambda^{2}-4 \mu} \xi}{2}\right)}{C_{1} \cosh \left(\frac{\sqrt{\lambda^{2}-4 \mu} \xi}{2}\right)+C_{2} \sinh \left(\frac{\sqrt{\lambda^{2}-4 \mu} \xi}{2}\right)}\right) \pm \frac{3 \lambda}{\sqrt{6 a}} i, \\
& \text { where } \xi=x-\frac{2-\lambda^{2}+4 \mu}{2} t .
\end{aligned}
$$

II. When $\lambda^{2}-4 \mu<0$, we have trigonometric function solution

$u_{2}(\xi)= \pm \sqrt{\frac{3\left(4 \mu-\lambda^{2}\right)}{2 a}} i\left(\frac{-C_{1} \sin \left(\frac{\sqrt{4 \mu-\lambda^{2}} \xi}{2}\right)+C_{2} \cos \left(\frac{\sqrt{4 \mu-\lambda^{2}} \xi}{2}\right)}{C_{1} \cos \left(\frac{\sqrt{4 \mu-\lambda^{2}} \xi}{2}\right)+C_{2} \sin \left(\frac{\sqrt{4 \mu-\lambda^{2}} \xi}{2}\right)}\right) \pm \frac{3 \lambda}{\sqrt{6 a}} i$ 
where $\xi=x-\frac{2-\lambda^{2}+4 \mu}{2} t$.

III. When $\lambda^{2}-4 \mu=0$, we get rational solution

$$
u_{3}(\mathrm{x}, \mathrm{t})= \pm \sqrt{\frac{6}{a}} i \frac{C_{2}}{C_{1}+C_{2}(x-t)} \pm \frac{3 \lambda}{\sqrt{6 a}} i \text {. }
$$

(I) The first set: If $\left(C_{1} \neq 0, C_{2}=0, \lambda>0, \mu=0\right)$ or $\left(C_{1}=0, C_{2} \neq 0, \lambda>0, \mu=0\right)$, then (4.9) gives

$$
\begin{aligned}
& u_{4}(\mathrm{x}, \mathrm{t})= \pm \sqrt{\frac{3}{2 a}} i \lambda \tanh \left[\frac{\lambda}{2}\left(x-\frac{2-\lambda^{2}}{2} t\right)\right] \pm \frac{3 \lambda}{\sqrt{6 a}} i \\
& u_{5}(\mathrm{x}, \mathrm{t})= \pm \sqrt{\frac{3}{2 a}} i \lambda \operatorname{coth}\left[\frac{\lambda}{2}\left(x-\frac{2-\lambda^{2}}{2} t\right)\right] \pm \frac{3 \lambda}{\sqrt{6 a}} i
\end{aligned}
$$

(II)

$$
\text { The }
$$

second

set:

$\left(C_{1} \neq 0, C_{2}=0, \lambda=0, \mu>0\right)$

$\left(C_{1}=0, C_{2} \neq 0, \lambda=0, \mu>0\right)$, then (4.10) becomes respectively

$$
\begin{gathered}
u_{6}(\mathrm{x}, \mathrm{t})=\mp \sqrt{\frac{6 \mu}{a}} i \tan [\sqrt{\mu}(x-(1+2 \mu) t)], \\
u_{7}(\mathrm{x}, \mathrm{t})=\mp \sqrt{\frac{6 \mu}{a}} i \cot [\sqrt{\mu}(x-(1+2 \mu) t)] .
\end{gathered}
$$

(III) The third set: If $\left(C_{1} \neq 0, C_{2}=0, \lambda=0, \mu<0\right)$ or $\left(C_{1}=0, C_{2} \neq 0, \lambda=0, \mu<0\right)$, then (4.9) gives respectively

$$
\begin{aligned}
& u_{8}(\mathrm{x}, \mathrm{t})=\mp \sqrt{\frac{6 \mu}{a}} \tanh [\sqrt{-\mu}(x-(1+2 \mu) t)], \\
& u_{9}(\mathrm{x}, \mathrm{t})= \pm \sqrt{\frac{6 \mu}{a}} \operatorname{coth}[\sqrt{-\mu}(x-(1+2 \mu) t)] .
\end{aligned}
$$

\section{Conclusions}

In this article, we investigated the BBM and the modified BBM equations using the generalized $\left(G^{\prime} / G\right.$ )-expansion method. This method is applied for finding travelling wave solutions of nonlinear evolution equations. This method has been successfully applied to obtain some new generalized solitonary solutions to the BBM and the MBBM equations. These exact solutions include three types hyperbolic function solution, trigonometric function solution and rational solution. The generalized $\left(\mathrm{G}^{\prime} / \mathrm{G}\right)$-expansion method is more powerful in searching for exact solutions of NLPDEs. Can be seen that the results are the same, with comparing results[24]. Also, new results are formally developed in this article. It can be concluded that the this method is a very powerful and efficient technique in finding exact solutions for wide classes of problems.that the this method is a very powerful and efficient technique in finding exact solutions for wide classes of problems.

\section{REFERENCES}

[1] Ablowitz, M.J. and P.A. Clarkson, 1991. Solitons, nonlinear evolution equations and inverse scattering, Cambridge: Cambridge University Press.

[2] Hirota, R., 2004. The Direct Method in Soliton Theory, Cambridge Univ. Press.

[3] Wazwaz, A. M., 2006. Travelling wave solutions for combined and double combined sine-cosine-Gordon equations by the variable separated ODE method, Appl. Math. Comput, 177: 755-760.

[4] Dehghan, M. and J. Manafian, 2009. The solution of the variable coefficients fourth-order parabolic partial differential equations by homotopy perturbation method, $\mathrm{Z}$. Naturforsch, 64: 420-430.

[5] Dehghan, M., J. Manafian and A. Saadatmandi, 2010. The solution of the linear fractional partial differential equations using the homotopy analysis method, Z. Naturforsch, 65a: 935-949.

[6] Dehghan, M., J. Manafian and A. Saadatmandi, 2010. Solving nonlinear fractional partial differential equations using the homotopy analysis method, Num. Meth. Partial Differential Eq. J, 26: 486-498.

[7] He, J. H., 1999. Variational iteration method a kind of non-linear analytical technique: some examples, Int. J. Nonlinear Mech, 34: 699-708.

[8] Dehghan, M., J. Manafian and A. Saadatmandi, 2010. Application of semi-analytic methods for the Fitzhugh-Nagumo equation, which models the transmission of nerve impulses, Math. Meth. Appl. Sci, 33: 1384-1398.

[9] Dehghan, M. and J. Manafian, 2011. Study of the wave-breaking's qualitative behavior of the Fornberg-Whitham equation via quasi-numeric approaches, Int. J. Num. Methods Heat Fluid Flow, to appear in 2011. scattering, Cambridge: Cambridge University Press.

[10] Fan, E., 2000. Extended tanh-function method and its applications to nonlinear equations, Phys. Lett. A, 277: 212-218.

[11] Menga, X. H., W. J. Liua, H. W. Zhua, C. Y. Zhang and B. Tian, 2008. Multi-soliton solutions and a Bäcklund transformation for a generalized variable-coefficient higher-order nonlinear Schrö dinger equation with symbolic computation, Phys. A, 387: 97-107.

[12] Manafian Heris, J. and M. Bagheri, 2010. Exact Solutions for the modified $\mathrm{KdV}$ and the Generalized $\mathrm{KdV}$ Equations via Exp-Function Method, J. Math. Extension, 4: 77-98.

[13] He, J. H., 2006. Non-perturbative method for strongly nonlinear problems. Dissertation, De-Verlag im Internet $\mathrm{GmbH}$, Berlin.

[14] He, J.H. and X.H. Wu, 2006. Exp-function method for nonlinear wave equations, Chaos, Solitons Fractals, 30: 700-708.

[15] Dehghan, M., J. Manafian and A. Saadatmandi, 2011. Application of the Exp-function method for solving a partial 
differential equation arising in biology and population genetics, Int. J. Num. Methods Heat and Fluid Flow, 21: 736-753.

[16] Dehghan, M., J. Manafian and A. Saadatmandi, 2011. Analytical treatment of some partial differential equations arising in mathematical physics by using the Exp-function method, Int. J. Modern Physics, B, 25: 2965-2981.

[17] Wang, M., X. Li and J. Zhang, 2008. The (G'/G )-expansion method and travelling wave solutions of nonlinear evolution equations in mathematical physics, Phys. Lett. A, 372: 417-423.

[18] Zhang, J., X. Wei and Y. Lu, 2008. A generalized $\left(\mathrm{G}^{\prime} / \mathrm{G}\right.$ )-expansion method and its applications evolution equations in mathematical physics, Phys. Lett. A, 372: $3653-3658$.

[19] Zhang, S., J. L. Tong and W. Wang, 2008. A generalized $\left(\mathrm{G}^{\prime} / \mathrm{G}\right)$-expansion method for the $\mathrm{mKdV}$ equation with variable coefficients, Phys. Lett. A, 372: 3653-3658.

[20] Bekir, A., 2008. Application of the ( $\left.\mathrm{G}^{\prime} / \mathrm{G}\right)$-expansion method for nonlinear evolution equations, Phys. Lett. A, 372:
3400-3406.

[21] Micu, S., 1999. On the controllability of the linearized Benjamin-Bona-Mahony equation, SIAM J Control Optim, 29: 1677-1696.

[22] Bona, J., 1981. On solitary waves and their role in the evolution of long waves. Applications of nonlinear analysis, Boston, MA: Pitman.

[23] Wazwaz, A. M., 2008. The extended tanh method for new compact and noncompact solutions for the KP-BBM and the ZK-BBM equations, Chaos Solitons Fractals, 38: 1505-1516.

[24] Yusofoğlu, E., 2008. New solitonary solutions for the MBBM equations using Exp-function method, Phys Lett A, 372: 442-446.

[25] Benjamin, T.B., J.L. Bona and J.J. Mahony, 1972. Philos. Trans. R. Soc. London, Ser. A, 272: 47-55.

[26] Saut, J.C. and N. Tzetkov, 2004. Appl. Math. Res. Express, 1: $1-11$.

[27] Varlamov, V. and Y. Liu, 2004. Discrete Dynam. Syst, 10: 731-739. 